\title{
Flicker vortex structures in multiferroic materials
}

\author{
Z. Zhao, ${ }^{1,2}$ X. Ding, ${ }^{2}$ and E. K. H. Salje ${ }^{1 *}$ \\ ${ }^{1}$ Department of Earth Sciences, University of Cambridge, Cambridge CB2 3EQ, UK \\ ${ }^{2}$ State Key Laboratory for Mechanical Behavior of Materials, Xi'an Jiaotong University, Xi'an \\ 710049, People's Republic of China \\ *Author to whom any correspondence should be addressed. \\ Email: ekhard@esc.cam.ac.uk
}

\begin{abstract}
Computer simulation of ferroelastic materials reveals dynamic polar vortex structures related to flexo-electricity between cation and anion lattices. At finite temperatures the vortices are found to flicker in time and space. Widely spaced ferroelastic twin boundaries nucleate vortices while dense twin boundaries suppress them. The time averaged number of vortices at any site decays exponentially indicating the highly mobile dynamics of the vortex lattice. Applied electric fields break the rotational symmetry of vortices and finally destroy them. The total number density of vortices follows a field and temperature dependence as $N(E)=N_{0} /[1+\mathrm{A} \exp (E / \mathrm{k}(T-T \mathrm{vF}))]$ with $T_{\mathrm{VF}}<0$. The observed vortex structures are akin to those observed in magnetic and superconducting disordered vortex lattices.
\end{abstract}

Key words: Vortex; Multiferroicity; Twinning; Disordered systems; non-equilibrium dynamics 
Evans at al. ${ }^{1}$ investigated the magnetic switching of ferroelectric domains in multiferroic PZTFT at room temperature and questioned the coupling mechanism between electric and magnetic degrees of freedom. They enumerated several aspects of their experimental observations, which are suggestive of a strain-mediated coupling: first, the magnetic fields cause changes in the ferroelastic, and not simply the ferroelectric, domain components of the microstructure; second, some amount of reversibility in domain reorientation has been observed while the magnetic fields often cause sporadic and unpredicted changes in domain states which are commensurate with sudden releases of elastic energy; third, the order of magnitude of the coupling coefficient is comparable to those found in heterostructures where strain-mediated coupling is deliberately targeted, possibly through selection of piezoelectric-magnetostrictive materials combinations. ${ }^{2}$ Similar sudden changes of polar and non-polar parameters are well known in disordered materials, where they follow power law distributions with all the characteristics of avalanche dynamics. ${ }^{3}$ Avalanches form when ferroelastic nanostructures change under fields and jam. ${ }^{4}$ Vortex structures have also been experimentally observed in wall-conducting $\mathrm{BiFeO}_{3}{ }^{5}$

No general mechanism has been proposed which could explain why some materials show simultaneously avalanche dynamics (jerks), polarity, and vortices. In this paper we follow the previous observation from computer simulations which shows that ferroelastic twin walls may become locally polar ${ }^{6-9}$ via an effective flexo-electric coupling between charged sublattices. The symmetry compatibility of polarity in twin walls was already described first in the pioneering papers by Janovec and collaborators. ${ }^{10}$ We then argue that polar vectors arrange themselves on a mesoscopic scale as vortex structures. In recent work, similar static vortex structures in ferroelectric materials have been seen in phase field simulations when flexo-electric coupling is explicitly included in the interactions. ${ }^{11}$ In this paper we will show that the implicit flexo-effect of charged sublattices leads to a different phenomenon which may also explain the avalanche 
dynamics of polar materials. We show that polar vortices are stable in number but unstable in their position: they flicker.

The generic model consists of two sublattices with positive and negative charges (Fig. 1). The atoms of the ferroelastic sublattice have negative elementary charges $\left(-1.602 \times 10^{-19} \mathrm{Clb}\right)$ while the second sublattice has positive elementary charges. Both sublattices have identical numbers of atoms to ensure electric neutrality. Long range Coulomb interactions and short range interatomic potentials govern the interaction between the two sublattices. We calculate the Coulomb interactions in the Ewald construction with a dielectric constant of 1000 which is typical for the incipient ferroelectric materials. Short range interatomic potentials are designed for the two sublattices separately, they mimic the chemical bonds. The interactions of ferroelastic sublattice consist of three components: (1) harmonic nearest neighbor interactions, $\mathrm{U}(\mathbf{r})=20(\mathbf{r}-1)^{2}$, (black springs in Fig. 1), (2) double well potentials for next nearest neighbors, $\mathrm{U}(\mathbf{r})=-10(\mathbf{r}-\sqrt{2})^{2}+2000(\mathbf{r}-\sqrt{2})^{4} \quad($ gray sticks along diagonals in Fig. 1); and fourth order interactions between the third nearest neighbors, $\mathrm{U}(\mathbf{r})=(\mathbf{r}-2)^{4}$ where $\mathbf{r}$ is the distance vector in units of angstrom. Double well potentials between next nearest neighbors were designed with the inspiration from Landau potentials to form a $4^{\circ}$ shear angle for the ferroelastic sublattice. These non-convex interactions reduce the symmetry of the square or cubic unit cell to monoclinic and hence generate spontaneous twinning. The second sublattice is placed inside the large cavities of the ferroelastic sublattice. The interactions among the second sublattice contain two convex components: harmonic nearest-neighbor springs (the same as in the ferroelastic sublattice, green springs in Fig. 1) and 6th order springs between the second nearest-neighbors, $\mathrm{U}(\mathbf{r})=3000(\mathbf{r}-\sqrt{2})^{6}$. These convex potentials stabilise the rectangular sublattice. Both sublattices are coupled by harmonic springs, $U(\mathbf{r})=0.1\left(\mathbf{r}-\frac{\sqrt{2}}{2}\right)^{2}$, and by Coulomb forces between the 
cations and anions.

The initial configuration is a two-dimensional sandwich with two pre-existing horizontal domain boundaries. The phase transition and microstructure of the ferroelastic structure is essentially the same if simulated in two or three dimensions, we profit here from the better statistical averaging in the two-dimensional structure. ${ }^{12}$ The typical geometry was $10 \mathrm{~nm} \times 10 \mathrm{~nm}$ and time steps of 0.005 ps. Periodic boundary conditions are adopted to avoid surface effects. The system was first relaxed using a conjugate gradient method to find the optimal position for each lattice point. Each configuration was then annealed at a given temperature between $1 \mathrm{~K}$ and $150 \mathrm{~K}$ for $2500 \mathrm{ps}$. An electric field was applied perpendicular to the initial twin boundaries after annealing at temperatures. The simulations were performed using the computer code LAMMPS. ${ }^{13}$ NPT ensemble was used in the isothermal and isobaric simulations. The temperature of the sample was held constant by the Nosé-Hoover thermostat.

The macroscopic spontaneous strain is determined by the macroscopic shear angle of the bulk after annealing. It decreases with increasing temperature and shows a ferroelastic phase transition at $T_{\mathrm{C}}=100 \mathrm{~K}$ (Fig. 2). The ferroelastic twin walls remain at their initial positions after annealing at low temperatures. Spontaneous nucleation of additional twin boundaries occurs near the transition point. During heating, these additional twin boundaries appear at $0.9 T_{\mathrm{C}}($ Fig. 3) the microstructure then evolves into cross-hatch tweed structures ${ }^{14}$ at temperatures above $T_{\mathrm{C}}$.

Dipoles form spontaneously and organise themselves on a mesoscopic scale. The most common pattern involves vortices which are recognised by the collective rotation of dipoles on a scale of some $1 \mathrm{~nm}$. In the middle of a vortex is a core with a diameter of 5 lattice constants. The vortex strength is calculated as the integral of the absolute dipole displacement inside the core. Vortices 
do not form static lattices in our simulations but are highly mobile (Fig. 3(a)) and 'flicker' in the same way as tweed nanostructures flicker in ferroelastic structures. ${ }^{14}$ The total number of vortices also fluctuates with time, its number density is Gaussian distributed. The system contains very few vortices at very low temperatures where thermal excitation is weak (Fig. 3(b)). With increasing temperature, the number of vortices increases dramatically and reaches saturation. A singularity was found in the phase transition temperature interval (Fig. 3(b)). Widely spaced twin boundaries nucleate vortices while high dense twin boundaries suppress vortices. The averaged vortex strength is dominated by thermal excitation but is enhanced in the transition temperature interval when the dipoles inside the twin boundaries contribute to vortices (Fig. 3(b)).

The number of vortices decays exponentially with time at any site (Fig. 4(a)) when the dipoles are time-averaged because vortices nucleate, move, and decay. The decay exponent is constant at low temperatures and reaches a minimum near the transition temperature because the polarity in the additional twin boundaries increase thermal disorder and hence increase the flicker of the vortices (Fig. 4(b)). The number of vortices is almost unchanged at high temperatures above $T_{\mathrm{C}}$ where dynamical tweed structures are formed.

The field dependence of the vortex structure was measured using electric fields perpendicular to the twin walls (Y direction in Fig. 3(a)). The fields break the rotational symmetry of vortices and finally destroy them. The number of vortices decays with increasing electric field strength $E$ (shown in Fig. 5). This decay follows $N=N_{0} /\left[1+\operatorname{Aexp}\left(E / E_{0}\right)\right]$ where $N_{0}$ is the number of vortices without electric field and $E_{0}$ is proportional to $T-T_{\mathrm{VF}}$ with $T_{\mathrm{VF}}=-0.45 T_{\mathrm{C}}$. This leads to an effective incipient Vogel-Fulcher behaviour where the energy $\mathrm{E}_{0}$ reaches zero at negative temperatures. Such behavior is not unusual for the transition of incipient ferroelectrics such as 
$\mathrm{SrTiO}_{3}$ where the extrapolated ferroelectric transition occurs at negative temperatures and where the glass freezing temperature is also negative. ${ }^{15}$

The simulated vortex structures explain avalanche behaviour (jerks) by jamming ${ }^{12}$ and show that strain induced coupling with polar properties is rather straightforward for twinned microstructures. The jerks are the same as simulated already for the ferroelastic lattice under strain deformation. In addition, the rapid movement and the field induced destruction of vortices also couple to the strain variable and can be observed by acoustic emission and dielectric measurements. Skyrmion lattices with magnetic vortex structures ${ }^{16}$ are similar to our polar vortex structures so that coupling between dipolar vortices and magnetic vortices becomes possible and may explain the origin of electric-magnetic coupling via a common strain. Superconducting vortex lattices follow a similar pattern with no long range order ${ }^{17}$ and also show jerky large scale oscillations under external fields. ${ }^{18}$ Direct observations of vortices in multiferroic materials are not uncommon (e.g. $\mathrm{YMnO}_{3}$ in Ref. 19) so that we believe that the gradient (flexo-) effects discussed in this paper are universal and a rather common phenomenon in multiferroic materials while their direct observation still remains elusive.

Acknowledgements: E.K.H.S. is grateful to EPSRC (RG66344) and the Leverhulme Foundation (RG66640) for support. Z.Z. and X.D. are grateful to NSFC (51171140, 51321003, 51320105014) for financial support. Z.Z. is supported by a scholarship from the China Scholarship Council. 
Figures:

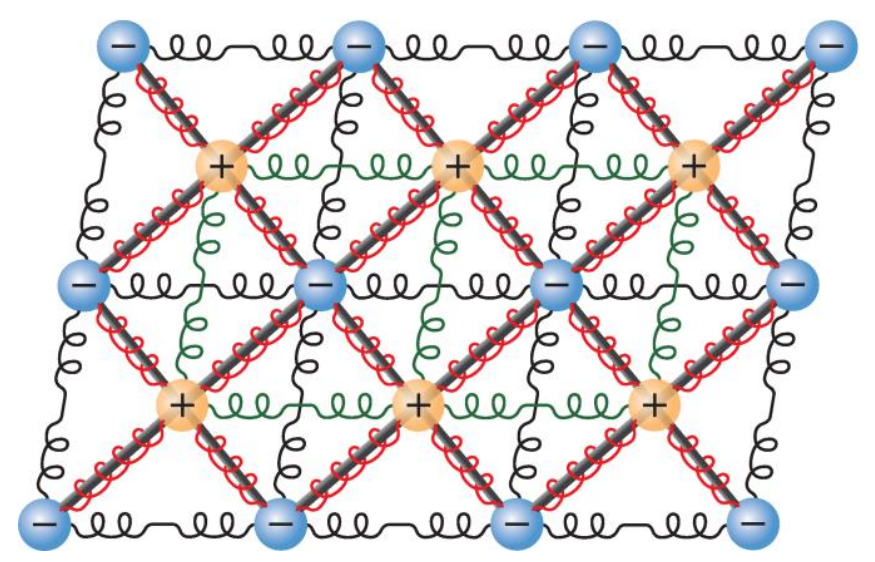

FIG. 1. Model with two charged sublattices. Coulomb interactions and interatomic interactions are combined in the model. The interatomic interactions between the nearest-neighbors are harmonic (shown by springs). Non-convex interactions (gray sticks) along diagonals in the ferroelastic sublattice lead to the formation of twin structures.

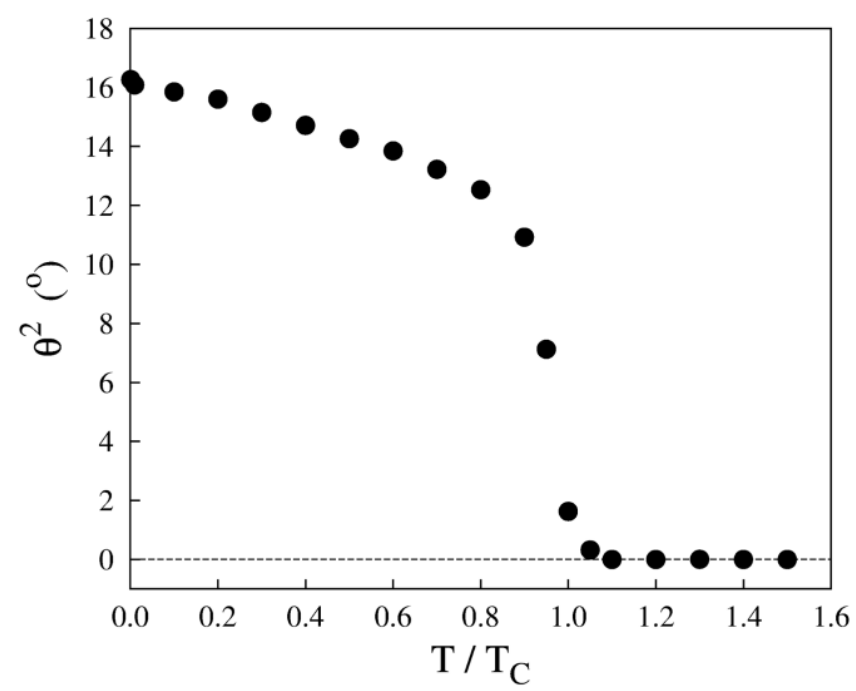

FIG. 2. Square of the macroscopic shear angle (proportional to the spontaneous strain) as function of temperature. The temperature scale is normalised by the ferroelastic phase transition temperature $T_{\mathrm{C}}=100 \mathrm{~K}$. 

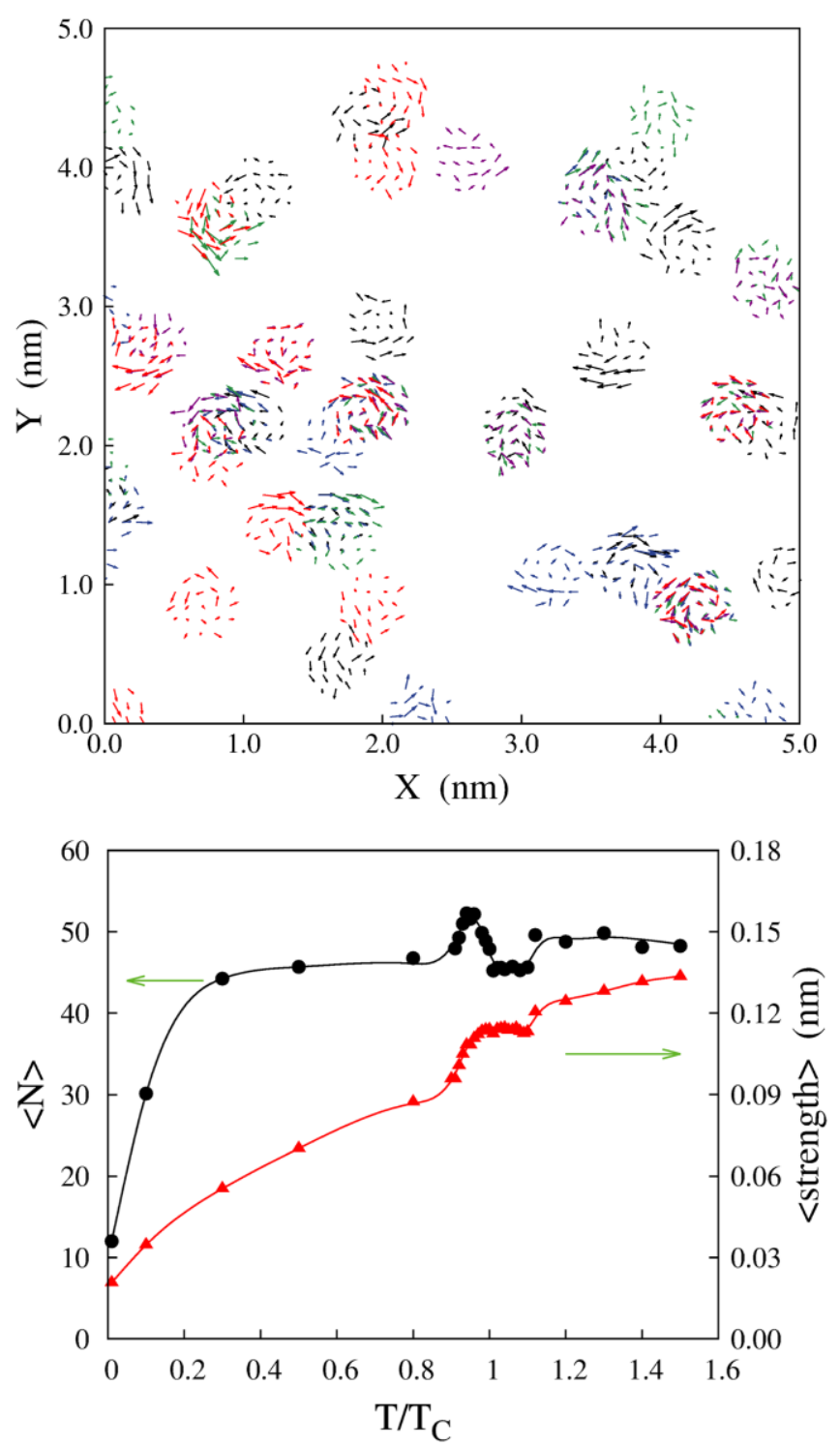

FIG. 3. Analysis of the observed vortex structures. (a) Vortices flicker at $0.9 T_{\mathrm{C}}$. Vortex structures are shown at $t_{0}+\Delta t$ (black), $t_{0}+2 \Delta t$ (blue), $t_{0}+3 \Delta t$ (green), $t_{0}+4 \Delta t$ (purple) and $t_{0}+5 \Delta t$ (red) where reference time $t_{0}=2500$ ps and time increment $\Delta t=0.05 \mathrm{ps}$. (b) Time averaged number of vortices $\langle N\rangle$ and vortex strength $\langle$ strength $\rangle$ variation with temperature. Singularity happens in the phase transition interval when additional twin boundaries nucleate. 

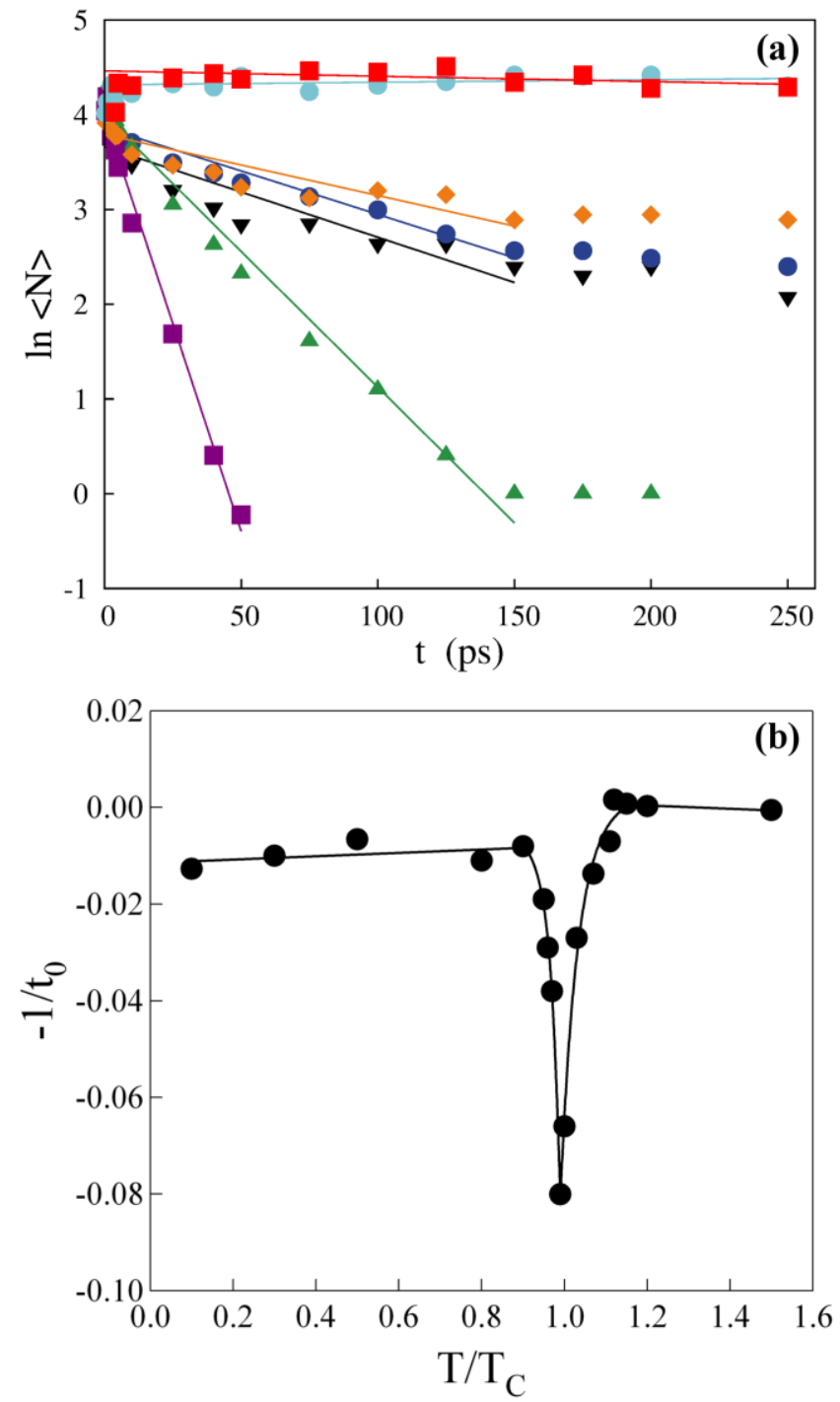

FIG. 4. (a) The number of vortices decays exponentially when the dipole movements are averaged over time. The decay follows $N \sim \exp \left(-t / t_{0}\right)$. Data at $0.3 T_{\mathrm{C}}$ (black triangles), $0.9 T_{\mathrm{C}}$ (dark blue circles), $0.96 T_{\mathrm{C}}$ (green triangles), $0.99 T_{\mathrm{C}}$ (purple squares), $1.1 T_{\mathrm{C}}$ (orange diamonds), $1.2 T_{\mathrm{C}}$ (light blue circles) and $1.5 T_{\mathrm{C}}$ (red squares) are shown in the graph. (b) The decay parameter $1 / t_{0}$ is constant at low temperatures and reaches a minimum in the transition temperature interval. The number of vortices is almost unchanged at temperatures above $T_{\mathrm{C}}$. with $1 / t_{0} \approx 0$. 


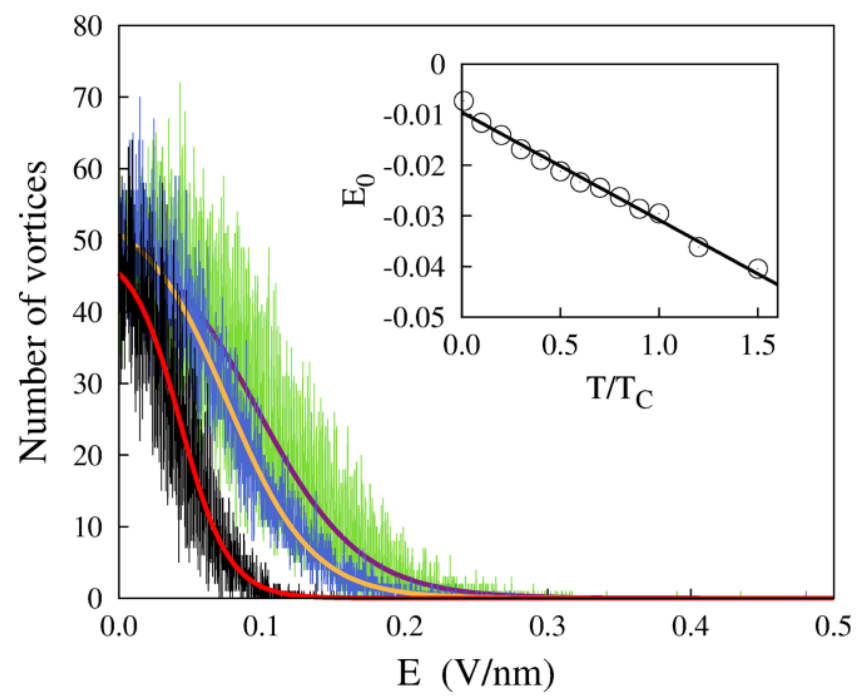

FIG. 5. The number of vortices decays with increasing electric field $E$. The decay follows Vogel-Fulcher type statistics $N_{0} /\left[1+\operatorname{Aexp}\left(E / E_{0}\right)\right]$ where $E_{0} \sim T-T_{\mathrm{VF}}$ shown in the inset graph. $N_{0}$ is the number of vortices without electric field and the extrapolated temperature $T_{\mathrm{VF}}=-0.45 T_{\mathrm{C}}$.

\section{References :}

${ }^{1}$ D. M. Evans, A. Schilling, A. Kumar, D. Sanchez, N. Ortega, M. Arredondo, R. S. Katiyar, J.

M. Gregg, and J. F. Scott, Nat. Commun. 4, 1534 (2013).

${ }^{2}$ T. H. E. Lahtinen, K. J. A. Franke, and S. van Dijken, Sci. Rep. 2, 258 (2012).

${ }^{3}$ E. K.H. Salje and K. A. Dahmen, Annu. Rev. Condens. Matter Phys. 5, 233 (2014).

${ }^{4}$ E. K. H. Salje, X. Ding, Z. Zhao, T. Lookman, A. Saxena, Phys. Rev. B 83, 104109 (2011).

${ }^{5}$ N. Balke, B. Winchester, W. Ren, Y. H. Chu, A. N. Morozovska, E. A. Eliseev, M. Huijben, R.

K. Vasudevan, P. Maksymovych, J. Britson, S. Jesse, I. Kornev, R. Ramesh, L. Bellaiche, L. Q.

Chen, and S. V. Kalinin, Nature Phys. 8, 81-88 (2012).

${ }^{6}$ S. Van Aert, S. Turner, R. Delville, D. Schryvers, G. Van Tendeloo, and E. K. H. Salje, Adv. Mater. 24, 523 (2012).

${ }^{7}$ L. Goncalves-Ferreira, S. A. T. Redfern, E. Artacho, and E. K. H. Salje, Phys. Rev. Lett. 101, 
097602 (2008).

${ }^{8}$ T. Zykova-Timan and E. K. H. Salje, Appl. Phys. Lett. 104, 082907 (2014).

${ }^{9}$ O. Aktas, E. K. H. Salje, M. A. Carpenter, V. V. Laguta, and J. F. Scott, Phys. Rev. Lett. 111, 247603 (2013).

${ }^{10}$ V. Janovec, Ferroelectrics 35, 105 (1981); J. Př́ivratská and V. Janovec, Ferroelectrics 222, 23 (1999); J. Přívratská, B. Shaparenko, V. Janovec, and D. B. Litvin, Ferroelectrics 269, 39 (2002).

${ }^{11}$ R. Ahluwalia, A. K. Tagantsev, P. Yudin, N. Setter, N. Ng, and D. J. Srolovitz, Phys. Rev. B 89, 174105 (2014).

${ }^{12}$ Z. Zhao, X. Ding, J. Sun, and E. K. H. Salje, J. Phys.: Condens. Matter 26, 142201 (2014).

${ }^{13}$ S. Plimpton, J. Comput. Phys. 117, 1 (1995).

${ }^{14}$ E. K. H. Salje, Annu. Rev. Mater. Res. 42, 265 (2012).

${ }^{15}$ A. V. Kityk, W. Schranz, P. Sondergeld, D. Havlik, E. K. H. Salje, and J. F. Scott, Phys. Rev. B 61, 946 (2000).

${ }^{16}$ X. Z. Yu, N. Kanazawa, Y. Onose, K. Kimoto, W. Z. Zhang, S. Ishiwata, Y. Matsui, and Y. Tokura, Nat. Mater. 10, 106 (2011)

${ }^{17}$ M. N. Chernodub, Phys. Rev. Lett. 106, 142003 (2011).

${ }^{18}$ L. Sochnikov, A. Shaulov, Y. Yeshurun, G. Logvenov, and I. Božović, Nat. Nanotechnol. 5, 516 (2010).

${ }^{19}$ S. C. Chae, Y. Horibe, D. Y. Jeong, S. Rodan, N Lee, and S.-W. Cheong, Proc. Natl. Acad. Sci. USA 107, 21366 (2010). 